JOURNAL OF SYNCHROTRON RADIATION

ISSN 1600-5775

Received 6 July 2015

Accepted 19 October 2015

Edited by D. Cocco, SLAC National Accelerator Laboratory, USA

Keywords: free-electron laser; detector; beam diagnosis; phosphors; pixelated phosphor detector.

\section{A novel approach in the free-electron laser diagnosis based on a pixelated phosphor detector}

\author{
Alessia Matruglio, ${ }^{\mathrm{a}, \mathrm{b}}$ Simone Dal Zilio, ${ }^{\mathrm{a} *}$ Rudi Sergo, ${ }^{\mathrm{c}}$ Riccardo Mincigrucci, ${ }^{\mathrm{d}, \mathrm{e}}$ \\ Cristian Svetina, ${ }^{b, f}$ Emiliano Principi, ${ }^{d}$ Nicola Mahne, ${ }^{f}$ Lorenzo Raimondi, ${ }^{f}$ \\ Alessio Turchet, ${ }^{\mathrm{g}}$ Claudio Masciovecchio, ${ }^{\mathrm{d}}$ Marco Lazzarino, ${ }^{\mathrm{a}}$ Giuseppe Cautero ${ }^{\mathrm{c}}$ \\ and Marco Zangrando ${ }^{\mathrm{a}, \mathrm{f}}$
}

\begin{abstract}
aOM Laboratorio Nazionale TASC, CNR, AREA Science Park Basovizza, SS 14, Km 163.5, Trieste 34149, Italy, ${ }^{\mathbf{b}}$ Graduate School in Nanotechnology, University of Trieste, Piazzale Europa 1, Trieste 34127, Italy, ' Detector and Instrumentation Laboratory, Elettra Sincrotrone Trieste, AREA Science Park Basovizza, SS 14, Km 163.5, Trieste 34149, Italy, ${ }^{\mathbf{d}}$ Beamlines Spectroscopy/Scattering, Elettra Sincrotrone Trieste, Area Science Park Basovizza, SS 14, Km 163.5, Trieste 34149, Italy, ' Dipartimento di Fisica e Geologia, Università degli Studi di Perugia, Via Alessandro Pascoli, Perugia 06123, Italy, ' Mechanical, Vacuum and Optical Engineering, Elettra Sincrotrone Trieste, Area Science Park Basovizza, SS 14, Km 163.5, Trieste 34149, Italy, and ${ }^{\mathbf{g}}$ Beamlines Microscopy/Diffraction, Elettra Sincrotrone Trieste, Area Science Park Basovizza, SS 14, Km 163.5, Trieste 34149, Italy. *Correspondence e-mail: dalzilio@iom.cnr.it
\end{abstract}

A new high-performance method for the free-electron laser (FEL) focused beam diagnosis has been successfully tested at the FERMI FEL in Trieste, Italy. The novel pixelated phosphor detector (PPD) consists of micrometric pixels produced by classical UV lithography and dry etching technique, fabricated on a silicon substrate, arranged in a hexagonal geometry and filled with suitable phosphors. It has been demonstrated that the overall resolution of the system has increased by reducing the diffusion of the light in the phosphors. Various types of PPD have been produced and tested, demonstrating a high resolution in the beam profile and the ability to measure the actual spot size shot-to-shot with an unprecedented resolution. For these reasons, the proposed detector could become a reference technique in the FEL diagnosis field.

\section{Introduction}

An essential prerequisite for experimental research using state-of-the-art free-electron laser (FEL) light sources is the proper characterization of the beam quality. Actually, the proper diagnosis in the field of pulsed high-power vacuum ultraviolet (VUV) and soft X-ray (SXR) sources is still an open issue. Up to now, several methods for determining transverse and longitudinal coherence (Allaria et al., 2012), spectra (Ayvazyan et al., 2002) and wavefront properties (Flöter et al., 2010) have been reported. However, only a few methods have been satisfactorily employed for the spatial profile of the beam characterization, such as: direct imaging at focus, employing YAG scintillators or phosphors screens (Tiedtke et al., 2009), ablative imprint over PMMA or silicon (Chalupský et al., 2007), or wavefront reconstruction by means of a wavefront sensor in the far field (Mimura et al., 2010). Each technique presents undeniable features for specific investigation. However, all of the techniques are also limited in other aspects (Chalupský et al., 2011): indentation provides the real spot size but is an ex situ technique and its analysis is very time consuming, even though some preliminary results show that this method could be extended in situ (Gerasimova et al., 2013); in contrast, the wavefront sensor provides realtime measurements but it works in a limited wavelength range and is an expensive technique; the application of screen 
scintillators allows real-time in situ imaging of the focused beam spot, making it one of the most diffused techniques; unfortunately they are easily saturated and the collected signal is degraded by the light-spreading phenomenon of emitted light, affecting the beam-shape analysis due to distortion of the collected image.

In the SXR and VUV fields, indirect digital imaging is needed and it is commonly obtained by coupling a scintillator film with a two-dimensional imaging sensor (CCD or CMOS devices). The problem in resolution and contrast derived from light spreading induced the implementation of a large variety of different strategies for performance enhancement: the logical improvement is the confinement of phosphors in individual neighbouring pixels reducing the optical cross-talk. For commercial screens, increased performance has been achieved by columnar or needle-like growth structures of CsI:Tl through thermal evaporation; however, their use in high-resolution imaging is limited for the not perfect guiding and confinement of light, resulting in 'cross-talk' between pixels (Nagarkar et al., 1998).

In our pixelated phosphor detector (PPD) devices, an array of micrometric phosphor pixels has been obtained by filling micrometric silicon pores arranged in a hexagonal geometry with suitable phosphor powders. Absorption studies on silicon (Green \& Keevers, 1995) confirm that, in the visible range, light diffusion is limited in the micrometre range; for this reason, bulk silicon seems an excellent material for our purpose. A similar approach has already been proposed in the literature: Svenonius et al. (2009) fabricated a structured scintillator filled by melting thallium-doped caesium iodide into a silicon pore array; the scintillator has been directly coupled to a CMOS detector allowing improved spatial resolution by exploiting the guiding effect of the $\mathrm{SiO}_{2} / \mathrm{Si}$ pore walls. Cha et al. (2011) employed evaporated CsI:Tl or $\mathrm{Gd}_{2} \mathrm{O}_{3}: \mathrm{Eu}$ deposited by a screen printing technique and obtained a similar pixelated screen. Coupling the device to a lens-coupled CCD imaging device, they demonstrated the effectiveness of the microstructured scintillators for high spatial resolution and improved contrast imaging.

Starting from similar concepts, we implemented a suitable pixelated phosphors technology for application in VUV and SXR FEL focused beam diagnosis. The proposed detector has been developed by a collaboration of IOM-CNR and FERMI@Elettra in the Italian Sincrotrone. The aim of the proposed PPD is to preserve the advantages of the scintillators (such as in situ and real-time detection), increasing their spatial resolution to achieve a reference technique for spatial quality diagnosis. In our setup, the direct coupling of the phosphors screen with the CCD camera was not possible due to the limits imposed by the geometries of the experimental vacuum chamber. Moreover, due to the high energy involved, the FEL beam is able to damage instantly every exposed material. For these reasons, uncoupled devices have been preferred: the beam imagining was achieved through a telemicroscope coupled with a CCD camera installed in the exterior of the vacuum chamber. Another aspect we considered for the design of our PPD is related to the requirement of higher resolution, closer to the beam size, of the order of a few micrometres; by reducing the pixels size in the range of a few micrometres or lower, the focused beam can be traced through the simple detection of the illuminated pixel phosphors in each cavity. At the moment, the focused beam size can then be determined by simply considering the pitch of the array and the lateral dimension of the cavity from where the light is emitted, which also defines the limit in the resolution of the system. The final result is a high-performance detector, able to measure the shot-to-shot actual spot size with unprecedented resolution for a classical phosphor screen. An interesting improvement of the PPD detector has been accomplished by coating the surface of the device with a thin film of indium tin oxide (ITO) and the first results obtained are presented in this article. Moreover, thanks to the simple design and fabrication process, the system can be easily modified and improved following the requirement of the FEL users.

\section{Materials and methods}

\subsection{PPD fabrication processes}

An array of micrometric holes arranged in a honeycomb geometry is fabricated in a clean silicon bulk substrate (500 $\mu \mathrm{m}$ thick) using a classical optical UV lithography technique. MEGAPOSIT ® SPR 2201.2 (SHIPLEY) photoresist has been patterned with holes with a diameter of $4 \mu \mathrm{m}$ and a distance between the pixels of $6 \mu \mathrm{m}$, with a total fill factor of active area of $70 \%$. A scheme of the array with its dimensions is shown in Fig. 1. The single device is $12 \mathrm{~mm} \times 12 \mathrm{~mm}$ and the patterned area is $10 \mathrm{~mm} \times 10 \mathrm{~mm}$. Dry etching in inductively coupled plasma-reactive ion etching has been performed exploiting the resist as the mask in order to transfer the pattern in the bulk silicon; an inductively coupled plasmaBosch-like process (Chang et al., 2005) (gases: $\mathrm{SF}_{6}, \mathrm{C}_{4} \mathrm{H}_{8}, \mathrm{Ar}$ ) has been set in order to obtain a depth of $5 \mu \mathrm{m}$, followed by $\mathrm{O}_{2}$ plasma so as to remove the resist mask. A scanning electron microscopy (SEM) image of the array of holes is shown in

Figure 1

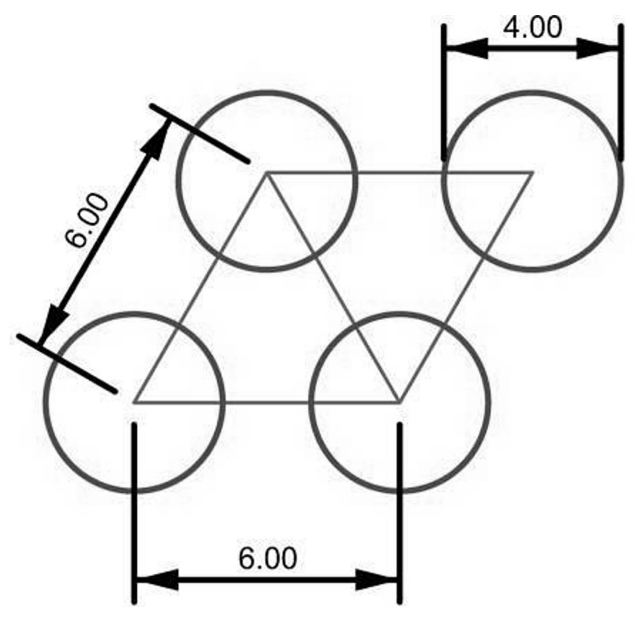

Scheme of the proposed array of micrometric holes arranged in a honeycomb geometry. The lengths are given in $\mu \mathrm{m}$. 
Table 1

Phosphors deposited and principal characteristics.

All phosphors were purchased from the Phosphor Technology Company.

\begin{tabular}{|c|c|c|c|c|c|}
\hline \multirow[b]{2}{*}{ Type } & \multirow[b]{2}{*}{ Composition } & \multirow[b]{2}{*}{$\begin{array}{l}\text { Maximum light } \\
\text { emission at: }\end{array}$} & \multirow[b]{2}{*}{ Colour } & \multicolumn{2}{|c|}{$\begin{array}{l}\text { Average grain size } \\
\text { of powder }\end{array}$} \\
\hline & & & & $\begin{array}{l}\text { Minimum } \\
(\mu \mathrm{m})\end{array}$ & $\begin{array}{l}\text { Maximum } \\
(\mu \mathrm{m})\end{array}$ \\
\hline P20 & $(\mathrm{Zn}, \mathrm{Cd}) \mathrm{S}: \mathrm{Ag}$ & $560 \mathrm{~nm}$ & Yellow, green & 1.2 & 6 \\
\hline P43 & $\mathrm{Gd}_{2} \mathrm{O}_{2} \mathrm{~S}$ & $560 \mathrm{~nm}$ & Green & 1.2 & 6 \\
\hline P46 & $\mathrm{Y}_{3} \mathrm{Al}_{5} \mathrm{O}_{12}: \mathrm{Ce}$ & $544 \mathrm{~nm}$ & Blue, white & 1.2 & 6 \\
\hline P47 & $\mathrm{Y}_{3} \mathrm{SiO}_{5}: \mathrm{Ce}, \mathrm{Tb}$ & $400 \mathrm{~nm}$ & Blue & 1.2 & 6 \\
\hline
\end{tabular}

Fig. 2(a). The deposition of four different types of commercially available phosphors (Phosphor Technology Company) has been achieved; the list of the powders and their corresponding characteristics are shown in Table 1 . The process of deposition of the phosphor powders has been accomplished by mechanical filling of the holes using a custom-developed piezoelectric vibrating plate; the vibration induces the alignment of the phosphor grains and helps the powder enter the micrometric holes. The dry technique allows the uniform and complete filling of the pores and avoids the trapping of air bubbles in the presence of solvent due to the surface tension of the liquid. A Teflon tip can be used during the process in order to help with the filling of the cavities. The excess of phosphor, in particular the grains with size larger than the pore size, is removed from the surface by the doctor blade

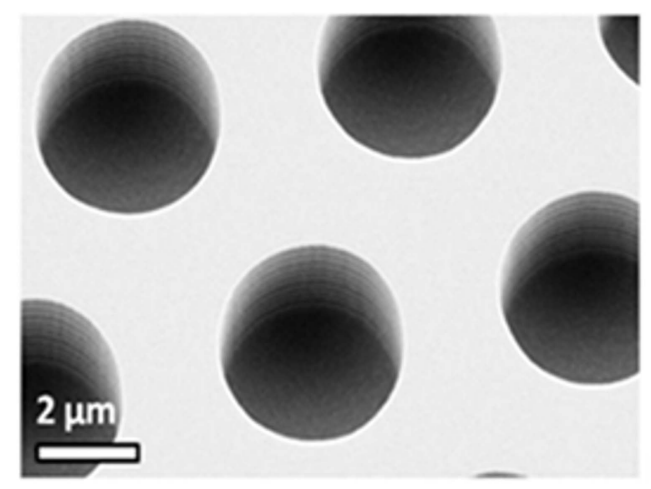

(a)

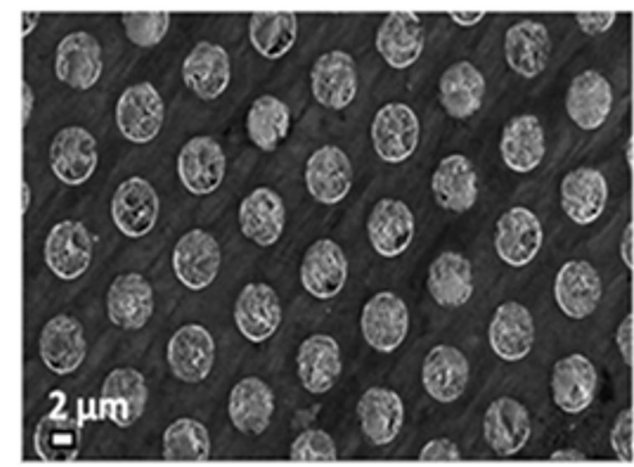

(b)

Figure 2

SEM images of (a) patterned silicon substrate and $(b)$ holes filled with P46 phosphors. No significant damage has been observed on the silicon substrate after the fabrication process.

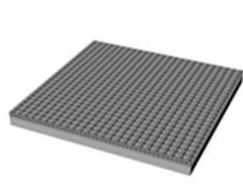

(a)

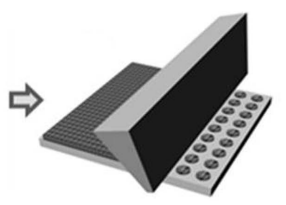

(b)

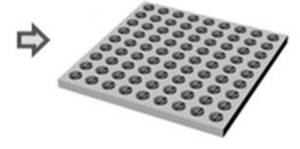

(c)
Figure 3

Scheme of the process of phosphor deposition: (a) sample coated with phosphors after the mechanical filling: phosphors are aligned in the cavities and are also present on the surface of the sample; $(b)$ removal of excess phosphor by the doctor blade technique; (c) final PPD with holes completely filled with phosphors.

technique (Yen et al., 2007) using a thick (1 mm) Teflon foil. As shown in Fig. 2(b), no significant damage has been observed on the silicon substrate after the followed protocol. A scheme of the process of phosphor deposition is shown in Fig. 3. Half of the samples have been processed in order to coat the PPD surface with a $100 \mathrm{~nm}$-thick film of ITO. The ITO film was deposited to prevent leakage of the phosphors powder from the holes and to limit the absorption of water and contaminants on the powders. A suitable magnetron sputtering process was implemented (power: $300 \mathrm{~W}$; pressure: 0.012 mbar; Ar flux: $25 \mathrm{sccm}$; sample temperature: $473 \mathrm{~K}$ ) in order not to damage the phosphors. A SEM image of the cross section obtained by a focused ion beam (FIB) of two pixels filled with P46 phosphors and coated with an ITO layer is shown in Fig. 4.

The homogeneity of the pixelated device has been investigated. Fig. 5 shows SEM images of two PPDs with P46 phosphors after FEL characterization, in the presence (Fig. 5a) and in the absence of an ITO film (Fig. 5b). A larger number of dark pixels can be observed in the second device. Higher magnification (insert in Fig. 5b) reveals a partial filling of the cavities, probably generated by a leak of phosphors; in contrast, the ITO-coated pixels look unaffected, in accordance with the hypothesis that the ITO coating acts as a barrier to the leakage of the phosphors from the silicon cavities.

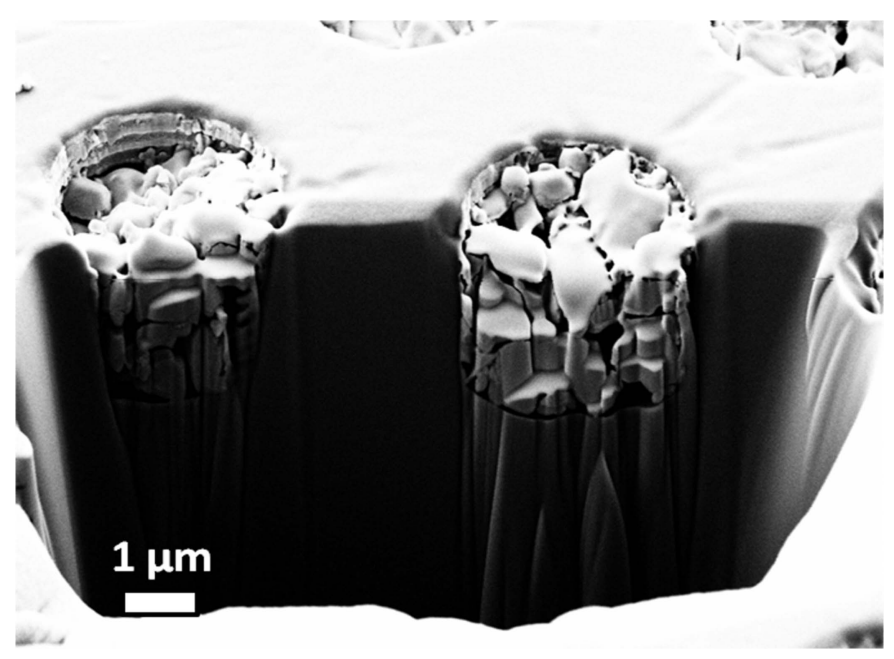

Figure 4

SEM image of the FIB cross section of two pixels covered with $100 \mathrm{~nm}$ of ITO layer. 


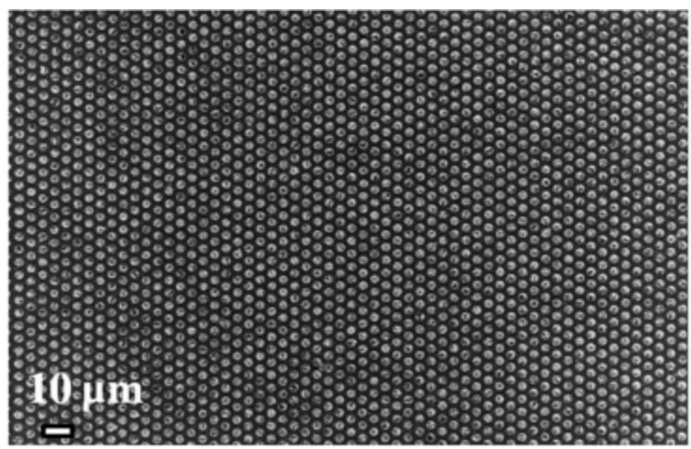

(a)

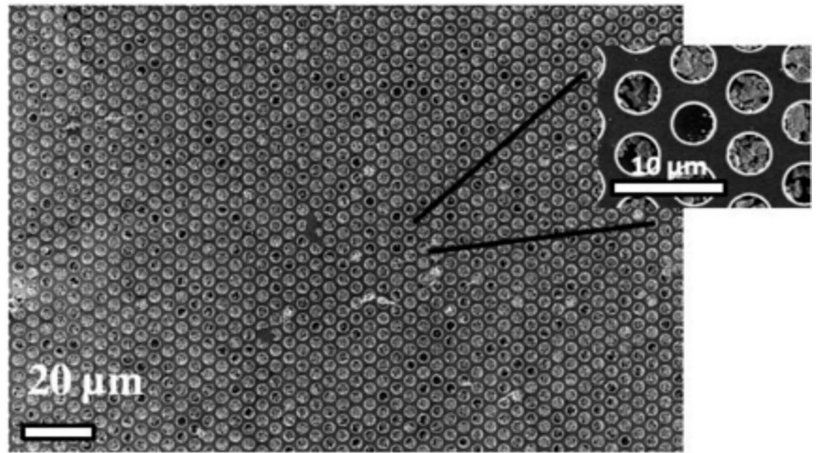

(b)

Figure 5

SEM images of PPD P46 filled after FEL measurements: $(a)$ with ITO coating; (b) without ITO coating; the insert in $(b)$ shows a magnification of the indicated area: partial filled cavities are visible.

\subsection{Experimental setup}

The experimental tests were performed at the EIS-TIMEX beamline (Masciovecchio et al., 2015) at the FERMI FEL in Trieste (Zangrando et al., 2014). The beamline optical design is characterized by an ellipsoidal focusing mirror of $1.4 \mathrm{~m}$ focal length. Phosphors fluorescence was detected with a telemicroscope, the Questar QM 100 long distance microscope, coupled to a visible CCD camera: Basler Scout scA640-74gm, monochromatic, 12-bit depth and dynamic range of 10.7 bit. The telemicroscope was placed $350 \mathrm{~mm}$ from the PPD at an angle of about $20^{\circ}$ with respect to the direction of the incoming FEL light. In this configuration, the resolution is better than $5 \mu \mathrm{m}$, the focal depth is a few tens of micrometres with a field of view about $600 \mu \mathrm{m}$. A scheme of the experimental setup is shown in Fig. 6. The telemicroscope calibration was performed before each measurement through the use of a micrometric scale, so the magnification is taken into account in every acquisition. A typical calibration achieved with the current set-up is of the order of 1000 pixels per $\mu \mathrm{m}$, which yields a resolution of $\sim 1 \mu \mathrm{m}$ per pixel, near to the optical resolution dictated by the telemicroscope itself.

\section{Results and discussion}

For the first set of measurements a PPD filled with P46 phosphors was used. For the present measurement campaign, the FEL was characterized by a pulse energy of $7 \mu \mathrm{J}$ and the wavelength used was $30.5 \mathrm{~nm}(40.7 \mathrm{eV})$. The intensity of the

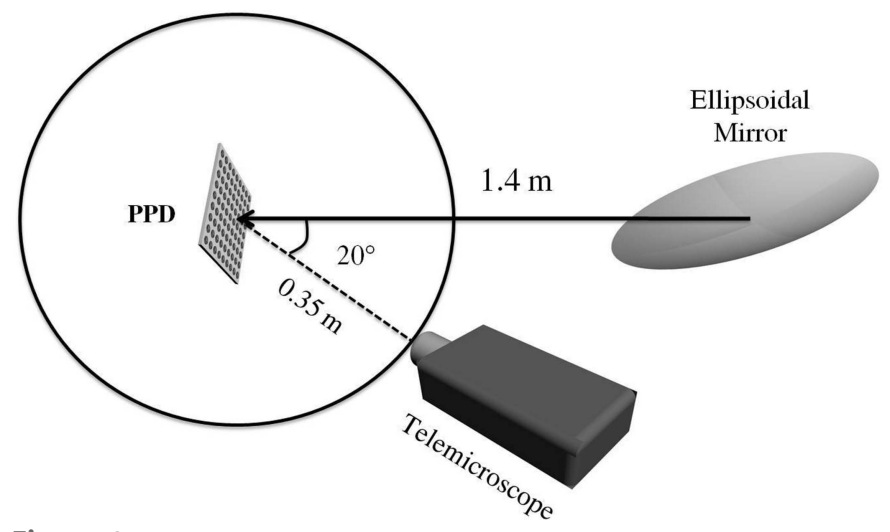

Figure 6

Scheme of the experimental setup of the EIS-TIMEX beamline.

even harmonics is zero for the in-axis emission, while the intensity of the third harmonic is below 1\% (Schmüser et al., 2008). Fig. 7( $a$ ) shows a CCD image of the PPD irradiated by ten shots of the photon beam; the CCD camera was operated at a frame rate of $10 \mathrm{~Hz}$, so the integration of ten shots was achieved by an exposure of $1 \mathrm{~s}$. Fig. 7(b) shows the same PPD after $\sim 10 \mathrm{~s}$ of exposure to the FEL radiation. Indeed, after $\sim 10 \mathrm{~s}$ of exposure, pixels lose intensity due to radiation damage, which causes ablation both of the substrate and of the phosphor pixels; studies on the damage threshold of the phosphors must be made to understand how many shots the phosphors are able to withstand. The direct observation of the light emitted by the illuminated pixels allows the size of the central spot to be estimated due to the fundamental harmonic: knowing the period of the lattice $(6 \mu \mathrm{m})$, supposing a single PPD pixel becomes illuminated, allows us to estimate that the central spot will be completely included in an area with a
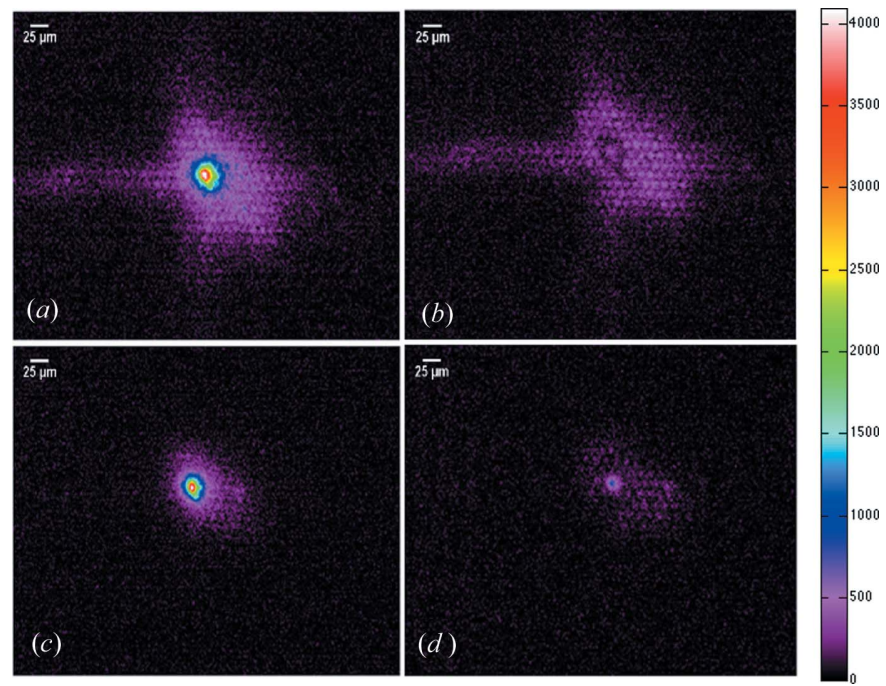

Figure 7

CCD camera image of the PPD with P46 phosphors hit with a pulse energy of $7 \mu \mathrm{J}$ at $30.5 \mathrm{~nm}$ wavelength; (a) integration of 10 FEL shots $(1 \mathrm{~s}$ of integration) on not coated PPD; (b) PPD after $\sim 10 \mathrm{~s}$ of exposure to the FEL beam; $(c)$ integration of 10 FEL shots ( $1 \mathrm{~s}$ of integration) on PPD ITO coated; $(d)$ PPD ITO coated after $\sim 10 \mathrm{~s}$ of exposure to the FEL beam. 

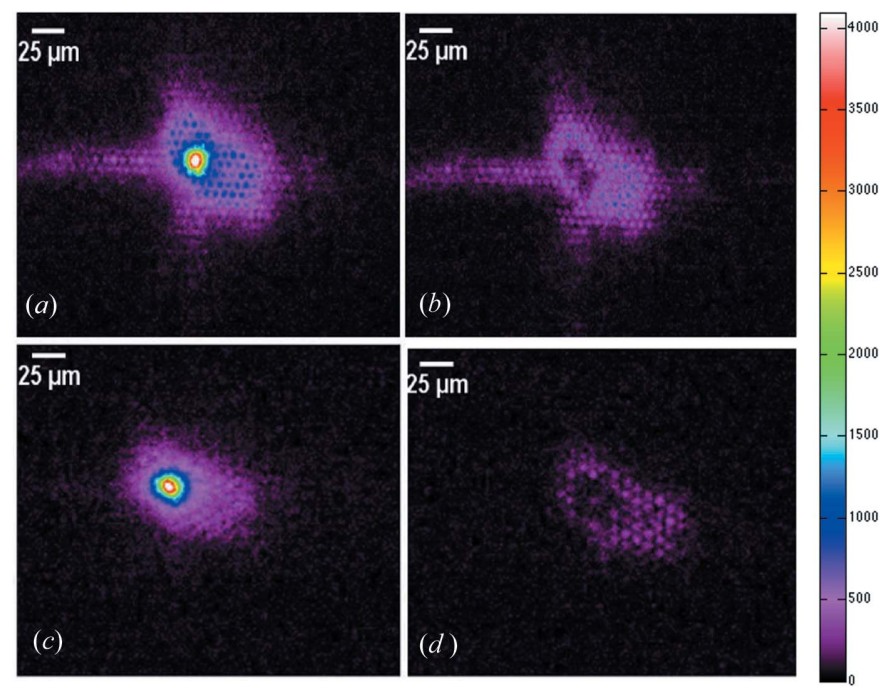

Figure 8

CCD camera image of the PPD with P47 phosphors hit with a pulse energy of $7 \mu \mathrm{J}$ and $30.5 \mathrm{~nm}$ wavelength; (a) integration of $10 \mathrm{FEL}$ shots ( $1 \mathrm{~s}$ of integration) on not coated PPD; (b) PPD after $\sim 10 \mathrm{~s}$ of exposure to the FEL beam; $(c)$ integration of 10 FEL shots (1 s of integration) on PPD ITO coated; (d) PPD ITO coated after $\sim 10 \mathrm{~s}$ of exposure to the FEL beam.

diameter of $8 \mu \mathrm{m}$ maximum. At the moment, a higher precision in the beam estimation cannot be provided due to the PPD resolution which is related to the size of the pixels and the characteristics of the telemicroscope. According to the specification, we know that the beam divergence is $50 \mu \mathrm{rad}$ and the expected spot size is $4.4 \mathrm{~mm}$ FWHM (Masciovecchio et al., 2015), in agreement with our measurements. In Figs. 7(c) and $7(d)$ measurements of ITO-coated PPDs are shown: it has been observed that the halo is weaker than in the case of the PPD without an ITO layer.

In the second set of measurements, a P47-phosphors-based PPD has been used. The experiment was performed on PPD coated and not coated with ITO. Fig. 8(a) shows measurements on a PPD with P47 phosphors without an ITO layer after $1 \mathrm{~s}$ of exposure to the photon beam, and Fig. 8(b) shows a PPD after $\sim 10 \mathrm{~s}$ of exposure; Fig. $8(c)$ shows a PPD with P47 phosphors ITO coated after $1 \mathrm{~s}$ of exposure and Fig. 8(d) shows it after $\sim 10 \mathrm{~s}$. Even the use of P47 allows the measurement of the size of the central beam spot (the limit in resolution is again $8 \mu \mathrm{m}$ ). As previously shown, the layer of ITO leads to a reduction of the halo.

In order to understand the absorption effect of the ITO layer, we have performed a characterization of the UV-Vis absorption of a film deposited under the same conditions of the capping layer on the PPDs. The measurements were carried out with a UV-Vis spectrometer on a $100 \mathrm{~nm}$-thick ITO film deposited on a glass slide. The spectrum (Fig. 9) shows that at the maximum emission wavelength of the P46 $(544 \mathrm{~nm})$ phosphor the transmission of the ITO is around $90 \%$, while for P47 (400 nm) phosphor it is almost $70 \%$. If the layer of ITO absorbs part of the radiation emitted by the phosphor, the halo on P46-filled PPD should be greater than that of the P47-filled PPD; because a slight dimming of the

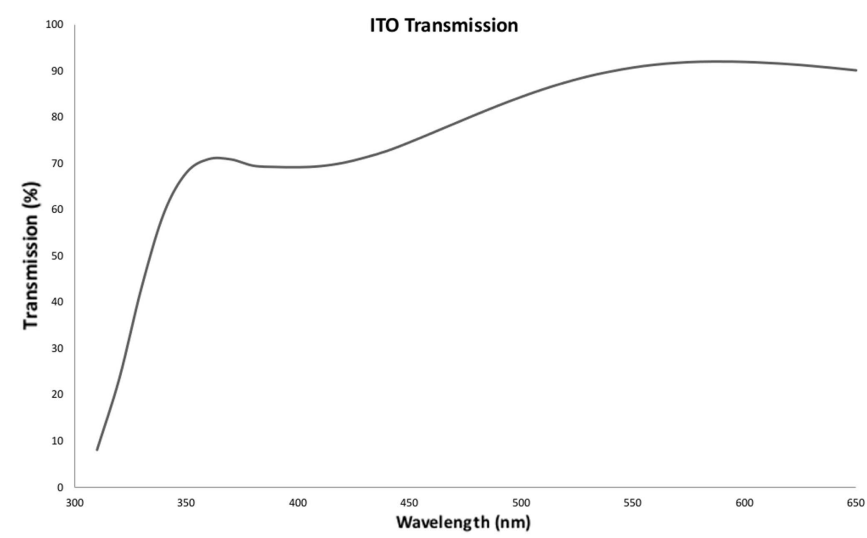

Figure 9

UV-Vis spectrum of a $100 \mathrm{~nm}$-thick ITO layer deposited on glass.

light is observed in the case of P46, we can conclude that the ITO cannot act as a filter for the light emitted by the phosphors. We can assume that the ITO could filter some components of the FEL beam, opening up the possibility of generating a selective filter directly in the screen detector just by changing the capping layer. Additional studies on ITO are essential in order to understand what harmonics are filtered and the mechanism of the process.

The last set of measurements has been performed to compare frosted P46 phosphor with a P46 phosphors PPD. Exposures were performed at a pulse energy of $1 \mu \mathrm{J}$ and a wavelength of $17 \mathrm{~nm}$ (energy $72.94 \mathrm{eV}$ ); the beam profile imaging is shown in Fig. 10. The difference in wavelength with respect to the previous measurements is due to a different tune of the machine, yet Fig. 10 suggests that the harmonic content is preserved. The present measurements were collected 'out of focus', but still allow us to derive important

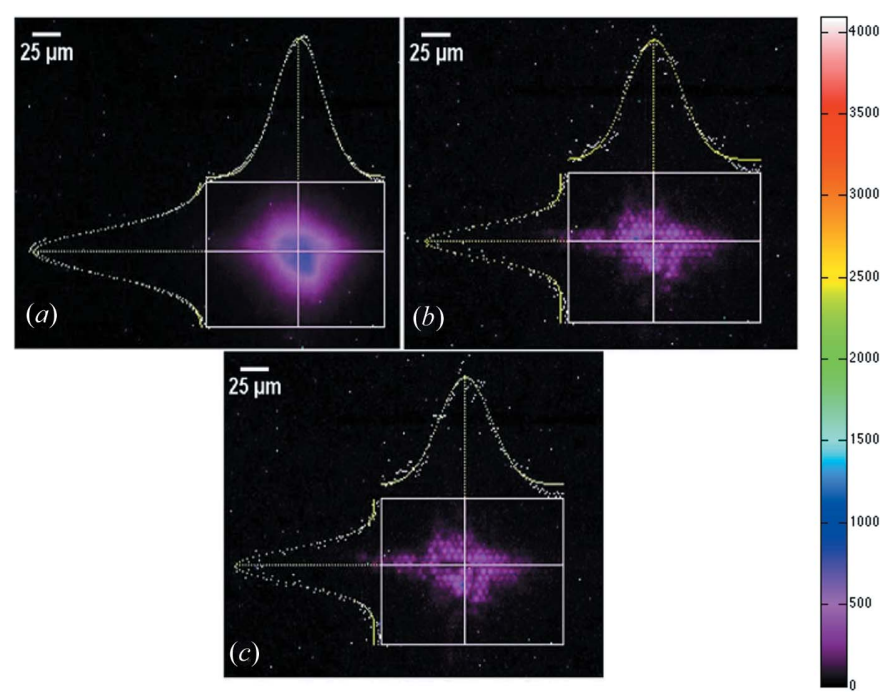

Figure 10

CCD camera images at $1 \mu \mathrm{J}$ pulse energy and $17 \mathrm{~nm}$ wavelength of: (a) frosted P46 phosphor after $1 \mathrm{~s}$ of exposure to the photon beam; measured spot size of horizontal r.m.s.: $46 \mu \mathrm{m}$; vertical r.m.s.: $37 \mu \mathrm{m}$; $(b)$ PPD P46 filled after $1 \mathrm{~s}$ of exposure to the photon beam; horizontal r.m.s.: $41 \mu \mathrm{m}$; vertical r.m.s.: $25 \mu \mathrm{m}$; $(c)$ PPD P46 filled after $30 \mathrm{~s}$ of exposure to the photon beam; horizontal r.m.s.: $46 \mu \mathrm{m}$; vertical r.m.s.: $28 \mu \mathrm{m}$. 
information such as the shape of the halo due to the other harmonics. The imaging with frosted P46 (Fig. 10a) is affected by the diffusion of emitted light in the phosphors: from the result it is impossible to clearly recognize the outlines of the halo due to higher-order harmonics. Indeed the PPD (Fig. 10b) allows us to accurately track the shape of the beam and to distinguish the fundamental harmonic from the other components. After $30 \mathrm{~s}$ of exposure to the beam (Fig. 10c) the radiation damage became evident, as in the previous experiments; nevertheless, the spatial imaging of the beam can still be acquired and characterized.

\section{Conclusions and outlooks}

A new beam detector has been produced and tested. A suitable and up-scalable fabrication process has produced a cost-effective and customizable device for FEL beam diagnosis through indirect imaging. Our devices demonstrated clear advantages when employed in high-flux beamlines (such as the EIS-TIMEX beamline in FERMI@ELETTRA FEL) that require the precise characterization of the focal spot to evaluate critical quantities such as the fluence on the sample. The PPD has demonstrated to be a powerful online tool for the beam diagnosis allowing superior spatial resolution in the imaging of the beam spot with respect to the YAG scintillators, remaining, at the same time, a real-time and in situ technique. Moreover, the regular structure will allow the implementation of an automatic focusing system for CCD cameras capable of moving the PPD in the optical axis of the focusing optics and measuring the spot size counting the number of the illuminated pixels. Every limitation owing to an incorrect visualization of the surface can be easily corrected due to the well known periodicity of the PPD surface itself.

We demonstrated that our system can be easily customized to use different phosphors in order to match the requirements of FEL wavelengths and decay time. Future development of the present work includes the tailoring of the composition and mixing of the phosphors deposited into the PPD. Higher resolution in the spot size measurement can be reached by changing the size of the holes (pixels), while by increasing the periodicity of the lattice it will be possible to improve the quality of the beam-shape evaluation.

The present measurement campaign suggests that the ITO coating could effectively work as a filter for some radiation components. A complete and detailed study of the role of the ITO coating has to be carried out to fully determine the spectral response of the coating itself. We plan to conduct absorption and transmission studies on the ITO layers with synchrotron radiation over a wide range of energy useful for FEL analysis.

A possible development of this kind of detector consists of making holes in a reflecting metal: this will direct all the phosphor-produced light toward the surface increasing the efficiency of PPD itself and making it more sensitive to weaker beams. For this reason, it could be possible to extend this imaging technique to other types of source such as synchrotron radiation.

\section{Acknowledgements}

The authors want to thank all colleagues of FERMI who made this work possible, in particular all the staff of EIS-TIMEX and DiProi and the FERMI Commissioning Team. A sincere thanks also to the Detectors \& Instrumentation laboratory staff and to the people of Istituto Officina dei Materiali. Thanks also to B. Marmiroli for help in the microfabrication. The work has partially founded by Italian Ministry of Education MIUR (FIRB RBAP11ETKA_003).

\section{References}

Allaria, E. et al. (2012). Nat. Photon. 6, 699-704.

Ayvazyan, V. et al. (2002). Phys. Rev. Lett. 88, 104802.

Cha, B. K., Lee, S. J., Muralidharan, P., Kim, D. K., Kim, J. Y., Cho, G., Jeon, S. \& Huh, Y. (2011). Nucl. Instrum. Methods Phys. Res. A, 652, 717-720.

Chalupský, J., Bohacek, P., Hajkova, V., Hau-Riege, S. P., Heimann, P. A., Juha, L., Krzywinski, J., Messerschmidt, M., Moeller, S. P., Nagler, B., Rowen, M., Schlotter, W. F., Swiggers, M. L. \& Turner, J. J. (2011). Nucl. Instrum. Methods Phys. Res. A, 631, 130-133. Chalupský, J. et al. (2007). Opt. Express, 15, 6036-6043.

Chang, C., Wang, Y.-F., Kanamori, Y., Shih, J.-J., Kawai, Y., Lee, C.-K., Wu, K.-C. \& Esashi, M. (2005). J. Micromech. Microeng. 15, 580585.

Flöter, B., Juranic, P., Kapitzki, S., Keitel, B., Mann, K., Plönjes, E., Schäfer, B. \& Tiedtke, K. (2010). New J. Phys. 12, 083015.

Gerasimova, N., Dziarzhytski, S., Weigelt, H., Chalupský, J., Hájková, V., Vyšín, L. \& Juha, L. (2013). Rev. Sci. Instrum. 84, 065104.

Green, M. A. \& Keevers, M. J. (1995). Prog. Photovolt. 3, 189-192.

Masciovecchio, C. et al. (2015). J. Synchrotron Rad. 22, 553-564.

Mimura, H., Handa, S., Kimura, T., Yumoto, H., Yamakawa, D., Yokoyama, H., Matsuyama, S., Inagaki, K., Yamamura, K., Sano, Y., Tamasaku, K., Nishino, Y., Yabashi, M., Ishikawa, T. \& Yamauchi, K. (2010). Nat. Phys. 6, 122-125.

Nagarkar, V. V., Gupta, T. K., Miller, S. R., Klugerman, Y., Squillante, M. R. \& Entine, G. (1998). IEEE Trans. Nucl. Sci. 45, 492-496.

Schmüser, P., Dohlus, M. \& Rossbach, J. (2008). Ultraviolet and Soft $X$-ray Free-Electron Lasers: Introduction to Physical Principles, Experimental Results, Technological Challenges, Springer Series Tracts in Modern Physics. Berlin: Springer.

Svenonius, O., Sahlholm, A., Wiklund, P. \& Linnros, J. (2009). Nucl. Instrum. Methods Phys. Res. A, 607 138-140.

Tiedtke, K. et al. (2009). New J. Phys. 11, 023029.

Yen, W. M., Shionoya, S. \& Yamamoto, H. (2007). Phosphor Handbook. Boca Raton: CRC Press/Taylor \& Francis.

Zangrando, M., Mahne, N., Raimondi, L. \& Svetina, C. (2014). Optical Technologies for Extreme Ultraviolet and Soft X-ray Coherent Sources, Springer Series in Optical Sciences, Vol. 197, edited by F. Canova and L. Poletto. Berlin: Springer. 